\title{
Design and analysis of a class of redundant collaborative manipulators with 2D large rotational angles
}

(C) The Author(s) 2019. This article is published with open access at link.springer.com and journal.hep.com.cn

\begin{abstract}
The parallel spindle heads with high rotational capability are demanded in the area of multi-axis machine tools and 3D printers. This paper focuses on designing a class of 2R1T (R: Rotation; T: Translation) parallel spindle heads and the corresponding collaborative 5-axis manipulators with 2-dimension (2D) large rotational angles. In order to construct 2D rotational degrees of freedom (DOFs), a platform with 2D revolute joints is proposed first. Based on the constraint screw theory, the feasible limbs that can be connected in the platform are synthesized. In order to provide constant rotational axis for the platform, a class of redundant limbs are designed. A class of redundant 2R1T parallel spindle heads is obtained by connecting the redundant limbs with the platform and the redundant characteristics are verified by the modified Grübler-Kutzbach criterion. The corresponding 5-axis collaborative manipulators are presented by constructing a 2-DOF series translational bottom moving platform. The inverse kinematics and the orientation workspace as well as the decoupling characteristics of this type of $2 \mathrm{R} 1 \mathrm{~T}$ parallel spindle heads are analyzed. The results show that these manipulators have large $2 \mathrm{D}$ rotational angles than the traditional A3/Z3 heads and can be potentially used in the application of multi-axis machine tools and the 3D printers.
\end{abstract}

Keywords parallel mechanism, redundant mechanism, large rotational angles, machine tools, 2R1T spindle head,

Received June 5, 2019; accepted August 28, 2019

Xiaodong JIN, Yuefa FANG $(\bowtie)$

Department of Mechanical Engineering, Beijing Jiaotong University, Beijing 100044, China

E-mail: yffang@bjtu.edu.cn

Xiaodong JIN, Dan ZHANG ( $ه)$, Xueling LUO

Department of Mechanical Engineering, Lassonde School of Engineering, York University, Toronto M3J 1P3, Canada

E-mail: dzhang99@yorku.ca collaborative manipulator

\section{Introduction}

The machine work and the additive manufacturing play a vital role in modern manufacturing industry. The parallel machine tools and 3D printers have the advantages of high speed, high precision, high rigidity, and high dynamic performance in comparison with their series counterparts. The most widespread parallel machine tools and 3D printers are 3-dimension translational type. If the print head only has three translational degrees of freedom (DOFs), as shown in Fig. 1(a), its trajectory is layer by layer and the modeling part is laminated by the printing materials. It will cause relatively severe step error in lateral surface of the workpiece. If non-horizontal parts (wedgeand arc-shaped as examples shown in Figs. 1(b) and 1(c)) need to be processed, it is a hard job for a pure translational manipulator whether it is cutting or $3 \mathrm{D}$ printing. As a result, the rotational DOF is demanded for the machining and 3D printing processes.

Some high-end machining centers have 5-axis or 6-axis process capability with 2 or 3 dimensional rotational DOFs [1]. However, most of the 5-axis and 6-axis machining centers at present suffer from a common disadvantage that relatively small rotational angles, which restricts the work performance. The famous 6-DOF Stewart-Gough platform and its derivative manipulators, which have been wellresearched and designed as simulators and industrial equipment, is hard to use in high-end situations because its moving platform can tilt no more than $40^{\circ}$ from the horizontal plane at the home position. Other traditional parallel mechanisms (PMs), such as 2-RPC/2-SPS [2], 3-RRCR [3], 3-PRPS [4], also suffer from the same problem due to the high kinematic coupling.

In comparison with the synthesis of the translational DOF, it is a more complex process for the synthesis of the rotational DOF. Very little literatures focus on the type 


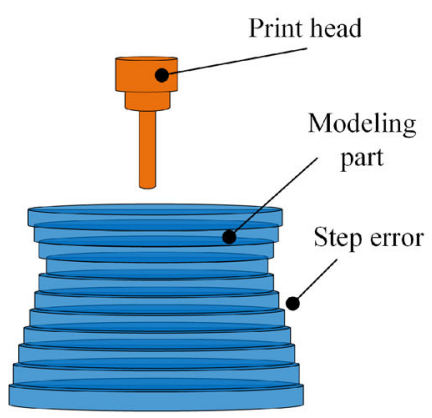

(a)

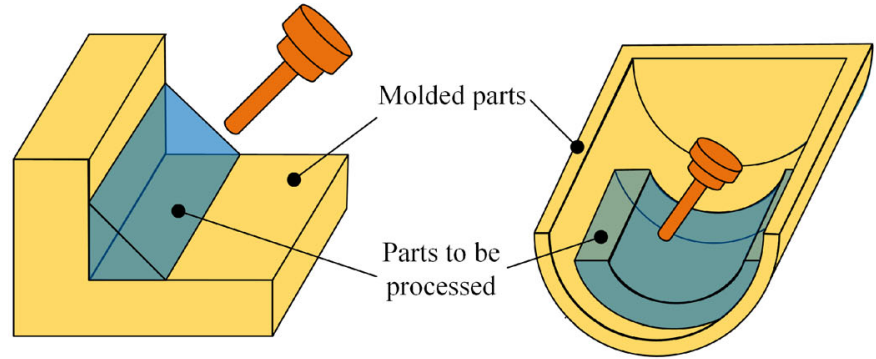

(b)

(c)

Fig. 1 Processing models: (a) 3-dimension translational 3D printing process; (b) wedge- and (c) arc-shaped workpiece.

synthesis about the PMs with large rotational angles, especially, with multi-dimensional large range rotations. The PM with pure rotational DOF is relatively easy to get large rotational angles, such as the 2-DOF Omni-Wrist III $[5,6]$ which has full $180^{\circ}$ hemisphere of yaw/pitch motion. However, the PM with both translational DOF and multidimension rotational DOF always suffer from lower rotational capability because of the interference. In order to design the PMs with high rotational performance, many researches have been done these years. The famous $\mathrm{H} 4$ family [7-9] is provided with large motion range through using a gear system and belt and pulley device to amplify the original rotation of the nacelle. Inspired by the $\mathrm{H} 4$ mechanisms, Guo et al. [10,11] proposed a class of 4-DOF (3-dimension translations and 1D rotation) PMs with large rotational workspace and presented a systematic method for the type synthesis of 4-DOF non-overconstrained PMs. To achieve multi-dimension high rotations, Wang et al. $[12,13]$ introduced the articulated platform to substitute for the traditional rigid platform. The articulated platform has the ability of rotating large angle benefits from the operation of arranging the rotation axes separated through two groups of orthogonal revolute joints for the platform in some sense. Hence, an essential task of designing PMs with multi-dimension rotational mobility is arranging the rotation axes in separate reasonably. Li et al. [14] classified the rotations into two types: If the line vectors are always through a specified point or line, the corresponding rotations are called continual rotations; otherwise, they are called non-continuous rotations.

Generally, the 5-DOF and 6-DOF PMs with high rotational capability have relatively complex limbs, which create more difficult kinematics, dynamics and control. Besides, the complex limbs also reduce the stability and increase the cumulative error of the mechanism. As a result, some researchers focus on developing 3-DOF 2R1T (R: Rotation; T: Translation) parallel spindle heads, and then connecting with the additional $2 \mathrm{D}$ translational parts to construct $2 \mathrm{R} 3 \mathrm{~T}$ collaborative manipulators $[15,16]$. The famous $\mathrm{Z3}$ spindle head [17] and A3 spindle head [18] are classic architectures which are built by the 3-PRS (R: Revolute joint; P: Prismatic joint; S: Spherical joint) and 3-RPS parallel structures respectively and both output 2R1T motion type. Numerous 2R1T PMs with or without parasitic motions have also been proposed [19-21]. Besides, the control method and strategy of 3RPS have also been studied [22]. Since spindle heads depending on the 3-RPS and 3-PRS suffer from lower rotational capability of less than $\pm 50^{\circ}$ and are difficult and costly to manufacture under the requirement of high precision and large rotational angles because of the spherical joints, they are limited to be used in the popular and low cost fields, such as desktop 3D printers. Thus, other researchers designed 2R1T spindle heads based on the 3-PUU (U: Universal joint) architecture $[23,24]$ to reduce the cost and increase the rotational workspace. In comparison with spherical joint and universal joint, the revolute joint has the advantage of low cost, easy to process and unlimited rotational capability. This paper is aimed at exploring high rotational 2R1T spindle heads and 2R3T collaborative manipulators whose rotations are realized by single-DOF kinematic pairs only, i.e., revolute joints and prismatic joints.

The methods for structural synthesis mainly include the enumeration approach based on the general GrüblerKutzbach mobility formula [25], the approach based on the screw theory [26-28], the synthesis approach based on the Lie group theory $[29,30]$, etc. This paper aims to design a class of 5-axis collaborative manipulators with 2D large rotational angles by means of the constraint screw theory. A class of parallel 2R1T spindle heads is presented based on the combination of the modified Grübler-Kutzbach criterion and constraint screw theory, in proposition of giving the rotational joints and axes associated with the moving platform. Then three collaborative manipulators are proposed by connecting the spindle heads and 2D translational mechanisms. Section 2 is basic conceptions and the moving platform with $2 \mathrm{D}$ revolute joints arrangement. Section 3 presents the 2R1T parallel spindle heads. Section 4 is the final collaborative manipulators. The kinematics and orientation performance are studied in Section 5. The conclusion is drawn in Section 6. 


\section{Basic conceptions and joints arrangement for moving platform}

\subsection{Basic conceptions}

\subsubsection{The modified Grübler-Kutzbach criterion}

According to Li et al. [31], the modified Grübler-Kutzbach criterion for spatial mechanisms can be written as

$$
M=d(n-g-1)+\sum_{i=1}^{g} f_{i}+v,
$$

where $M$ is the DOF of a mechanism, $d$ is the order of this mechanism, $n$ denotes the number of links that includes the base, $g$ is the number of joints, $f_{i}$ is the degrees of relative motion permitted by the $i$ th joints, and $v$ represents the number of overconstraints. Further, $d$ can be expressed as

$$
d=6-\lambda,
$$

where $\lambda$ denotes the number of the independent common constraints in the mechanism. A common constraint is defined as a wrench reciprocal to the unit twists associated with all kinematic pairs in a lower-mobility PM. In other words, the wrench system of each limb contains this common constraint.

\subsubsection{Screw theory and the constraints synthesis theory}

A screw is defined by

$$
\boldsymbol{S}=\left(\begin{array}{c}
\boldsymbol{s} \\
\boldsymbol{s}_{0}
\end{array}\right)= \begin{cases}\left(\begin{array}{c}
\boldsymbol{s} \\
\boldsymbol{r} \times \boldsymbol{s}+h \boldsymbol{s}
\end{array}\right) & \text { if } h \text { is finite, } \\
\left(\begin{array}{l}
\mathbf{0} \\
\boldsymbol{s}
\end{array}\right) & \text { if } h \text { is infinite, }\end{cases}
$$

where $\boldsymbol{s}$ is a direction vector of the screw axis, $\boldsymbol{r}$ is a position vector of any point of the screw axis with respect to the reference frame, and $h$ is the pitch of the screw. $\boldsymbol{s}_{0}=\boldsymbol{r} \times \boldsymbol{s}$ is the moment of the screw axis about the origin of the reference frame.

The screw is called a twist if it represents the instantaneous motion of a rigid body, and a wrench if it represents a system of forces and couples applied on a rigid body.

Two screws, $\$$ and $\$^{\mathrm{r}}$, are said to be reciprocal if they satisfy the condition:

$$
\boldsymbol{S} \circ \boldsymbol{S}^{\mathrm{r}}=0
$$

where

$$
\boldsymbol{S} \circ \boldsymbol{S}^{\mathrm{r}}=\boldsymbol{s} \cdot \boldsymbol{s}_{0}^{\mathrm{r}}+\boldsymbol{s}^{\mathrm{r}} \cdot \boldsymbol{s}_{0} .
$$

According to Fang et al. [32,33], a limb with lower- mobility that can provide a constraint force or a constraint couple is named as an F-limb (denoted by $\mathrm{L}_{\mathrm{F}}$ ) or a C-limb (denoted by $\mathrm{L}_{\mathrm{C}}$ ), and an limb that can provide both a constraint force and a constraint couple is named as an FC-limb, which is denoted by $\mathrm{L}_{\mathrm{FC}}$. The geometric conditions of axes for the prismatic and revolute joints of $\mathrm{L}_{\mathrm{F}}$ and $\mathrm{L}_{\mathrm{C}}$ are given by Fang et al. [32,33]:

C1. All the revolute joints of an F-limb must be intersecting or parallel to the constraint force.

C2. All the prismatic joint axes of an F-limb are perpendicular to the constraint force.

C3. All the revolute joint axes of a C-limb must be perpendicular to the constraint couple.

C4. The prismatic joint axes of a C-limb can be oriented arbitrarily.

These conditions are also suited for $\mathrm{L}_{\mathrm{FC}}$.

The number of DOF for an end-effector can be written as

$$
F=6-C,
$$

where $F$ denotes the output DOF of the moving platform, and $C$ is the number of independent constraints. In other words, $C$ denotes the order of the wrench system, and $F$ denotes the order of the twist system. It should be paid great attention that the mobility of a PM is different from the output DOF of the end-effector because there may be passive DOF in a PM, which results in the PM's DOF more than the output DOF.

\subsection{Synthesis procedure}

A common synthesis method based on the screw theory is the constraints synthesis theory, which usually only meets the constraints and DOF requirements of the PMs. In order to obtain the PMs with high rotational capability, the synthesis method proposed in this paper gives priority to the design of a moving platform with relatively independent rotation axes, which relies on single-DOF revolute joints. The remaining design process is also based on the constraints synthesis theory on the premise of guaranteeing the condition of relatively independent rotation axes. Since the moving platform with relatively independent rotation axes may bring passive DOF for the limbs, an indispensable procedure is to check the passive DOF in the limbs and add corresponding kinematic redundant limb to control the passive DOF if needed. The detailed synthesis procedure is shown in Fig. 2.

\subsection{Joints arrangement for moving platform}

The kinematic pairs that can provide rotational axes and enable the platform to rotate about these axes include the revolute joints, the screw joints, the cylindrical joints, the universal joints and the spherical joints. As passive translational DOF and concomitant motion in a PM should be avoided as much as possible, the screw joints and the 


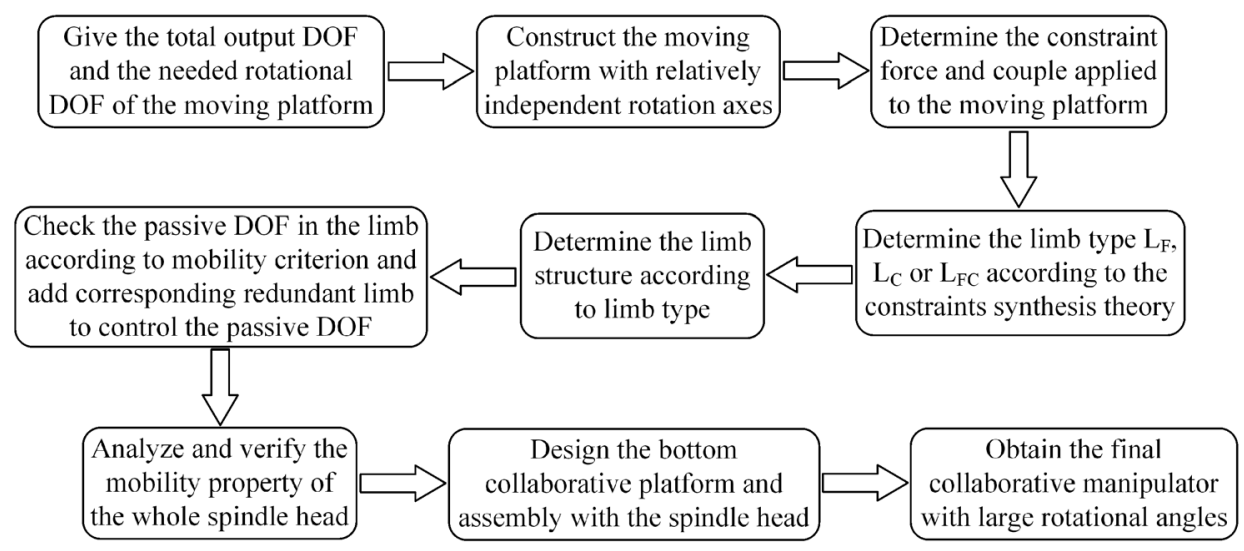

Fig. 2 Synthesis procedure flow.

cylindrical joints are not considered to provide the rotational axes. Besides, the universal joints and the spherical joints are not used in this paper as well due to their structural limited rotational range. As a result, only revolute joints are selected to construct the rotational axes for the platform, depending on their unlimited single-DOF rotational capability.

For a platform that output 2D rotational DOF with large angles, it basically needs a pair of orthogonal rotational axes or axis-cluster. First, we use three revolute joints $(B$, $C$, and $D$ shown in Fig. 3 ) to construct a set of rotational axis-cluster. Joints $C$ and $D$ are collinear and provide a rotational axis $\boldsymbol{v}_{1}$, and joint $B$, which is parallel to joints $C$ and $D$, provides a rotational axis $\boldsymbol{v}_{2}$. A rotational axiscluster is formed by $\boldsymbol{v}_{1}$ and $\boldsymbol{v}_{2}$, which means that the platform is able to rotate about any axis $\boldsymbol{v}$ that parallels to axes $\boldsymbol{v}_{1}$ and $\boldsymbol{v}_{2}$ within the workspace. Then an additional revolute joint $A$, whose axis $\boldsymbol{u}$ is parallel to the platform to provide the second rotational DOF about $x$-direction, is connected to joint $B$ and ought to keep parallel with $x$-axis constantly. The whole structure connected by the platform and joints $A, B, C$, and $D$ is represented by [PLA]. In summary, two critical steps in designing a $2 \mathrm{R} 1 \mathrm{~T}$ spindle

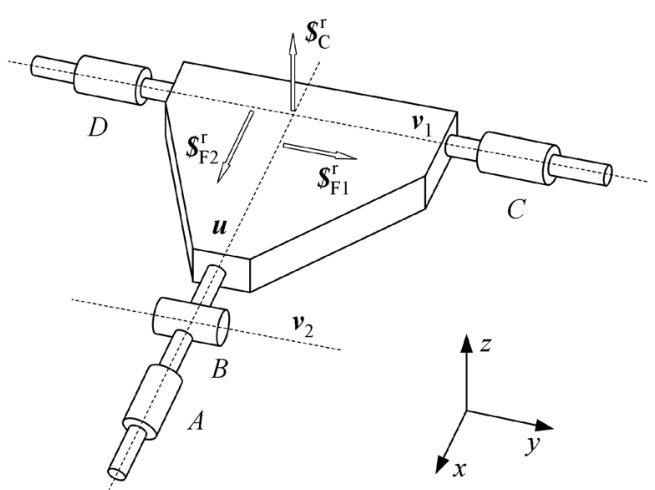

Fig. 3 [PLA] with 2D rotational axes. Subscripts $F$ and $C$ represent force and couple, respectively. head are to synthesize the limbs structure and to orient the axis of joint $A$. The limb connected to joints $A$ and $B$ is defined as limb 1 and the limbs connected to joints $C$ and $D$ are named as limb 2 and limb 3, respectively.

\section{The 2R1T spindle head and its mobility performance}

\subsection{Synthesis of the basic limb structures}

Considering the platform possesses two-dimension rotations about $x$ and $y$ directions, and the rotation about $z$-direction should be restricted, the wrench system of the moving platform should contain a constraint couple or several parallel constraint couples, which are parallel to $z$-axis. Since the maximum linearly independent number of the spatially parallel dual vectors is one, these parallel constraint couples should equivalent to a common constraint couple, which is denoted as $\boldsymbol{S}_{\mathrm{C}}^{\mathrm{r}}$, as shown in Fig. 3.

To obtain the required 2R1T PM, the wrench system should be a 3-order system, in which the other two orthogonal constraint forces are needed. To guarantee the limbs structure symmetry as much as possible, we let the limbs connected in the platform be $\mathrm{L}_{\mathrm{FC}}$, which is acted upon a constraint force and a constraint couple. Due to the fact that all the revolute joints in a $\mathrm{L}_{\mathrm{F}}$ must be parallel or intersecting to the constraint force, the position and direction of the constraint force in each limb should be intersecting with $\boldsymbol{u}$ or $\boldsymbol{v}$ and parallel to $\boldsymbol{v}$ or $\boldsymbol{u}$, respectively, as shown in Fig. 3, in which the constraint force is denoted as $\boldsymbol{S}_{\mathrm{Fi}}^{\mathrm{r}}$.

$$
\left\{\begin{array}{l}
\boldsymbol{S}_{\mathrm{C}}^{\mathrm{r}}=\left(\begin{array}{llllll}
0 & 0 & 0 ; & 0 & 0 & 1
\end{array}\right), \\
\boldsymbol{S}_{\mathrm{F} 1}^{\mathrm{r}}=\left(\begin{array}{llllll}
0 & 1 & 0 ; & -b & 0 & a
\end{array}\right) \\
\boldsymbol{S}_{\mathrm{F} 2}^{\mathrm{r}}=\left(\begin{array}{llllll}
1 & 0 & 0 ; & 0 & b & -a
\end{array}\right)
\end{array}\right.
$$

As mentioned before, $\boldsymbol{\$}_{\mathrm{C}}^{\mathrm{r}}$ is a common constraint, as a 
result, the wrench system of each limb should contain it. Besides, $\boldsymbol{S}_{\mathrm{F} 1}^{\mathrm{r}}$ should be contained in the wrench system of limb 1. $\boldsymbol{S}_{\mathrm{F} 2}^{\mathrm{r}}$ should be associated with the wrench system of limbs 2 and 3. Thus, the wrench system for limb 1 can be expressed as:

$$
\left\{\begin{array}{l}
\boldsymbol{S}_{\mathrm{C} 1}^{\mathrm{r}}=\left(\begin{array}{llllll}
0 & 0 & 0 ; & 0 & 0 & 1
\end{array}\right), \\
\boldsymbol{S}_{\mathrm{F} 1}^{\mathrm{r}}=\left(\begin{array}{llllll}
0 & 1 & 0 ; & -b & 0 & a
\end{array}\right) .
\end{array}\right.
$$

The wrench system associated with limbs 2 and 3 are

$$
\begin{gathered}
\left\{\begin{array}{l}
\boldsymbol{S}_{\mathrm{C} 2}^{\mathrm{r}}=\left(\begin{array}{llllll}
0 & 0 & 0 ; & 0 & 0 & 1
\end{array}\right), \\
\boldsymbol{S}_{\mathrm{F} 2}^{\mathrm{r}}=\left(\begin{array}{llllll}
1 & 0 & 0 ; & 0 & b & -a
\end{array}\right),
\end{array}\right. \\
\left\{\begin{array}{l}
\boldsymbol{S}_{\mathrm{C} 3}^{\mathrm{r}}=\left(\begin{array}{llllll}
0 & 0 & 0 ; & 0 & 0 & 1
\end{array}\right), \\
\boldsymbol{S}_{\mathrm{F} 3}^{\mathrm{r}}=\left(\begin{array}{llllll}
1 & 0 & 0 ; & 0 & b & -a
\end{array}\right) .
\end{array}\right.
\end{gathered}
$$

For this platform, a critical factor to guarantee the rotation about $x$-direction when the platform rotates about $y$-direction first is to maintain joint $A$ parallel to $x$. Thus, a passive DOF is much in evidence in limb 1 to orient the rotational axis. Besides, the twist system associated with limb 1 should contain a linearly dependent twist due to this passive DOF. Hence, the twist system of limb 1 is:

$$
\left\{\begin{array}{l}
\boldsymbol{S}_{11}=\left(\begin{array}{llllll}
0 & 0 & 0 ; & 1 & 0 & 0
\end{array}\right), \\
\boldsymbol{S}_{12}=\left(\begin{array}{llllll}
0 & 0 & 0 ; & 0 & 0 & 1
\end{array}\right), \\
\boldsymbol{S}_{13}=\left(\begin{array}{llllll}
0 & 1 & 0 ; & 0 & 0 & 0
\end{array}\right), \\
\boldsymbol{S}_{14}=\left(\begin{array}{llllll}
1 & 0 & 0 ; & 0 & b & 0
\end{array}\right), \\
\boldsymbol{S}_{15}=\alpha \boldsymbol{S}_{11}+\beta \boldsymbol{S}_{12}+\gamma \boldsymbol{S}_{13}
\end{array}\right.
$$

where $\$_{11}, \$_{12}, \$_{13}$, and $\$_{14}$ represent the reciprocal twist system related to Eq. (8), $\$_{15}$ denotes the twist associated with the passive DOF, and $\alpha, \beta$, and $\gamma$ are constants which are not equal to zero at the same time.

And the twists system associated with limbs 2 and 3 are

$$
\left\{\begin{array}{l}
\boldsymbol{S}_{i 1}=\left(\begin{array}{llllll}
0 & 0 & 0 ; & 0 & 1 & 0
\end{array}\right), \\
\boldsymbol{S}_{i 2}=\left(\begin{array}{llllll}
0 & 0 & 0 ; & 0 & 0 & 1
\end{array}\right), \\
\boldsymbol{S}_{i 3}=\left(\begin{array}{llllll}
1 & 0 & 0 ; & 0 & 0 & 0
\end{array}\right), \\
\boldsymbol{S}_{i 4}=\left(\begin{array}{llllll}
0 & 1 & 0 ; & -b & 0 & 0
\end{array}\right),
\end{array} \quad i=2 \text { and } 3 .\right.
$$

Based on Eqs. (11) and (12), $\$ \$_{11}$ denotes a prismatic joints $\mathrm{P} x$ along $x$-direction, $\$_{12}$ represents a prismatic joints $\mathrm{P} z$ along $z$-direction, $\$_{13}$ is a revolute joint $\mathrm{R} y$ around $y$-direction, and $\$_{14}$ and $\$_{15}$ are the twists associated with joints $A$ and $B$. As a result, $\mathrm{P} x \mathrm{P} z \mathrm{R} y$ is a theoretically qualified kinematic chain that can be connected with joint $A$ in Fig. 3. However, $\mathrm{P} x \mathrm{P} z \mathrm{R} y$ kinematic chain will bring a passive prismatic joint in the limb, which should be avoided as much as possible. A group of bases consist of $\$_{11}, \$_{12}$, and $\$_{13}$ is actually the screw bases of the planer motion, which is a $2 \mathrm{~T} 1 \mathrm{R}$ motion in a plane that represented by the linear combination of this bases. Specifically

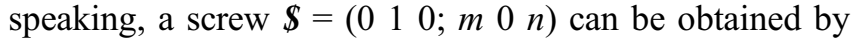
linear combination of $\$_{11}, \$_{12}$, and $\$_{13}$, and this screw denotes a revolute joint about $y$-direction, the same as $\$_{15}$. Hence, the kinematic chains that are equivalent to the motion characteristics of $\mathrm{P} x \mathrm{P} z \mathrm{R} y$ and without passive prismatic joints are $\mathrm{R} y \mathrm{R} y \mathrm{R} y, \mathrm{P} z \mathrm{R} y \mathrm{R} y$, and $\mathrm{R} y \mathrm{P} z \mathrm{R} y$. Similarly, the kinematic chains connected in joints $C$ and $D$ in [PLA] can be obtained as $\mathrm{R} x \mathrm{R} x \mathrm{R} x, \mathrm{P} z \mathrm{R} x \mathrm{R} x$, and $\mathrm{R} x \mathrm{P} z \mathrm{R} x$. As a result, three basic parallel spindle head structures with the same motion characteristics can be obtained after connecting the kinematic chains with [PLA], that are $3 \dot{R} \dot{R} \dot{R}-[P L A], 3 P \dot{R} \dot{R}-[P L A], 3 \dot{R} P \dot{R}-[P L A]$, where the dots above the symbols $\mathrm{R}$ are used to distinguish the orientation of the revolute joints within a limb. An example structure $3 \dot{R} \dot{R} \dot{R}-[P L A]$ is illustrated in Fig. 4.

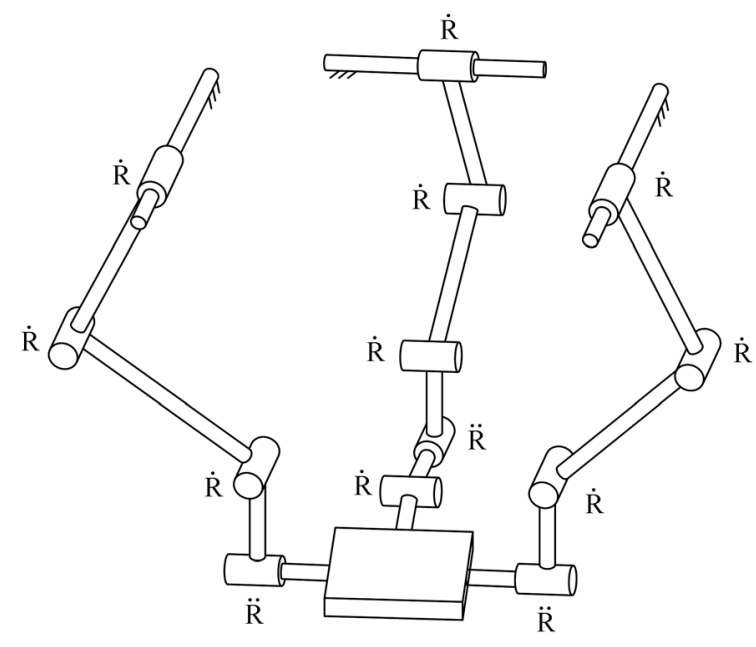

Fig. 4 An example structure $3 \dot{R} \dot{R} \dot{R}-[P L A]$ that satisfies the constraint condition.

\subsection{Orienting of joint $A$}

The first step that constructing the basic limb structures to satisfy the instantaneous constraint and mobility requirements is completed. However, joint $A$ in [PLA] cannot be oriented if only one actuator is installed in the base of each limb because the passive DOF $\$_{15}$ is added in limb 1 .

Based on Eq. (7), there is one common constraint $\boldsymbol{S}_{\mathrm{C}}^{\mathrm{r}}$ at the initial posture, which result in $d=5$. From Eqs. (9) and (10), there exists an overconstraint $\boldsymbol{S}_{\mathrm{F} 3}^{\mathrm{r}}$, as a result, $v=1$. Hence, DOF of these PMs in the initial position can be calculated out:

$$
\begin{aligned}
M & =d(n-g-1)+\sum_{i=1}^{g} f_{i}+v \\
& =5 \times(12-13-1)+13+1=4 .
\end{aligned}
$$


And DOF of the end-effector is

$$
F=6-C=6-3=3 .
$$

$M>F$, which proves there is a passive DOF in each $\mathrm{PM}$. To control this passive DOF, three redundant kinematic chains, whose structures are same as the kinematic chains connected in joint $A$ proposed in Section 3.1, are constructed to connect with joint $A$. For example, for the $3 \dot{R} \dot{R} \dot{R}-[\mathrm{PLA}]$, an additional kinematic chain $\dot{\mathrm{R}} \dot{R} \dot{\mathrm{R}}$ is connected to joint $A$ to control the passive DOF. The two kinematic chains connected in joint $A$ form a local closedloop kinematic chain in a limb and a new hybrid limb is built in view of the whole limb. As a result, a new parallel 2R1T spindle head $4 \dot{R} \dot{R} \dot{R}$-[PLA] with a redundant limb that enable the orienting capability of joint $A$ is obtained as illustrated in Fig. 5(a). Similarly, the other two basic parallel spindle head structures $3 \mathrm{PR} \dot{\mathrm{R}}$-[PLA] and $3 \dot{R} P \dot{R}$ [PLA] can be evolved into 4PRं $\dot{R}-[\mathrm{PLA}]$ and $4 \dot{\mathrm{R} P \dot{R}}$ [PLA], respectively, as shown in Figs. 5(b) and 5(c).

\subsection{Mobility analysis}

The limbs 2 and 3 are isostructural, as shown in Fig. 6(a). Its constraints applied in the platform are constant no matter what posture the platform is. According to the conceptions in Section 2.1.2, $\boldsymbol{\$}_{\mathrm{C} 2}^{\mathrm{r}}$ is always perpendicular to the plane determined by the axes of joints $D, E, F$, and $G$, and $\boldsymbol{S}_{\mathrm{F} 2}^{\mathrm{r}}$ is constantly located in this plane and perpendicular and intersect to the axis of joint $D$.

However, limb 1 is a hybrid limb, which is consisted of a parallel closed loop chain $L M N-P Q R$ and two series revolute pairs $A B$, as shown in Fig. 6(b). The constraints applied in the platform are variable with changing of the platform's posture.

The twist system associated with the parallel part $L M N-$ $P Q R$ of limb 1 can be written as:

$$
\left\{\begin{array}{l}
\boldsymbol{S}_{11}=\left(\begin{array}{llllll}
0 & 1 & 0 ; & 0 & 0 & 0
\end{array}\right), \\
\boldsymbol{S}_{12}=\left(\begin{array}{llllll}
0 & 1 & 0 ; & -b_{1} & 0 & a_{1}
\end{array}\right), \\
\boldsymbol{S}_{13}=\left(\begin{array}{llllll}
0 & 1 & 0 ; & -b_{2} & 0 & a_{2}
\end{array}\right), \\
\boldsymbol{S}_{11}^{a}=\left(\begin{array}{llllll}
0 & 1 & 0 ; & 0 & 0 & 0
\end{array}\right), \\
\boldsymbol{S}_{12}^{a}=\left(\begin{array}{llllll}
0 & 1 & 0 ; & -b_{3} & 0 & a_{3}
\end{array}\right), \\
\boldsymbol{S}_{13}^{a}=\left(\begin{array}{llllll}
0 & 1 & 0 ; & -b_{4} & 0 & a_{4}
\end{array}\right) .
\end{array}\right.
$$

Thus, the standard base of the wrench system of this part can be deduced out:

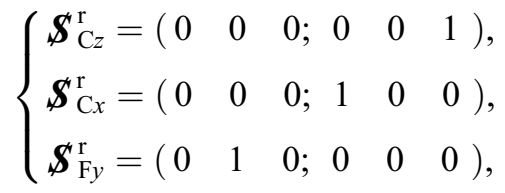

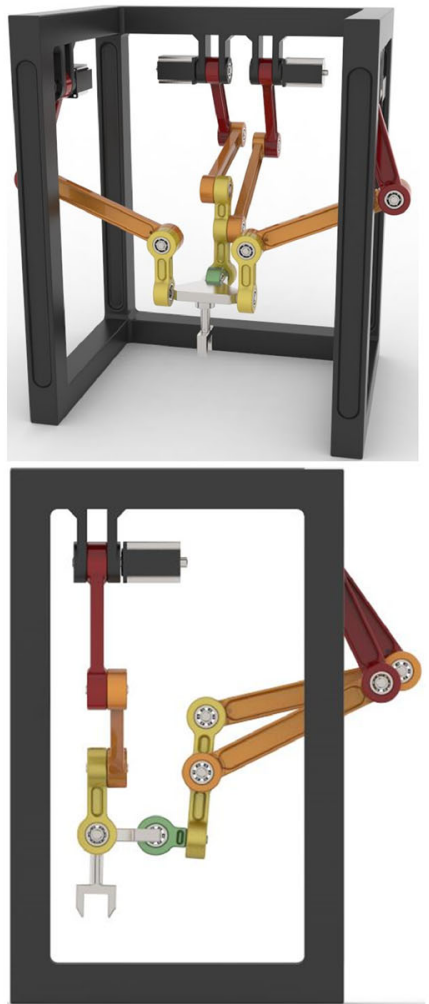

(a)

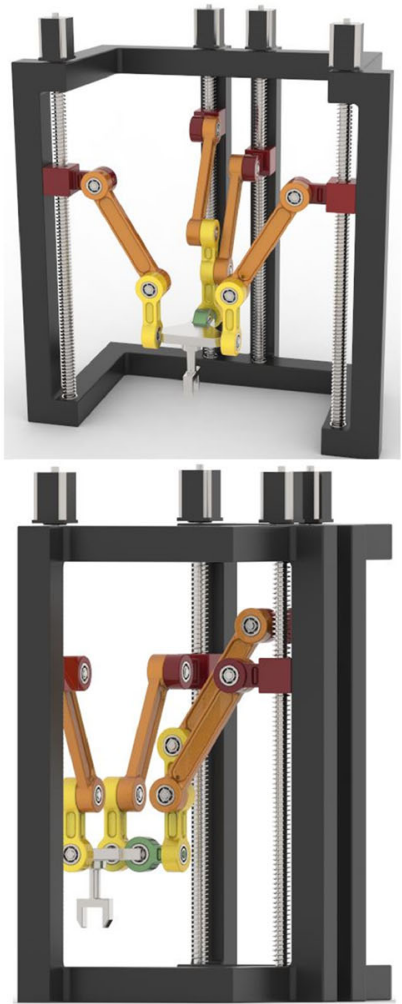

(b)

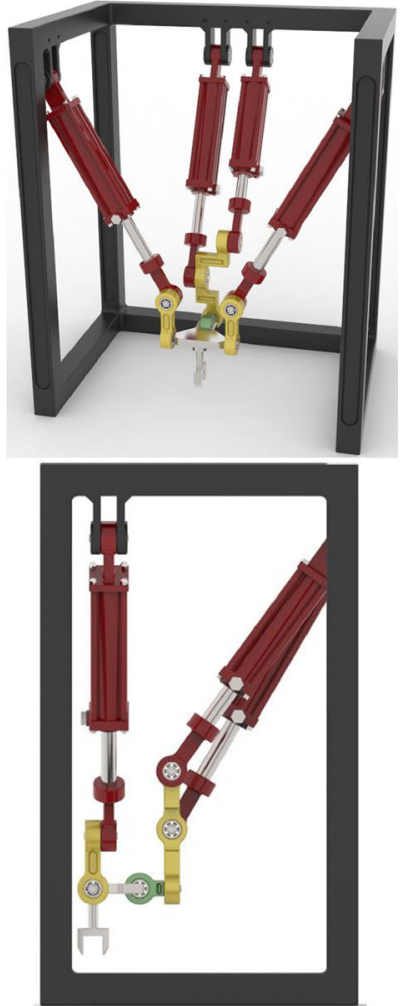

(c)

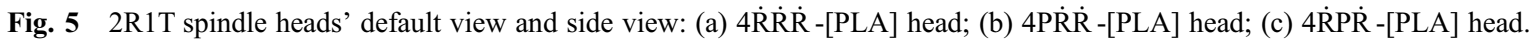




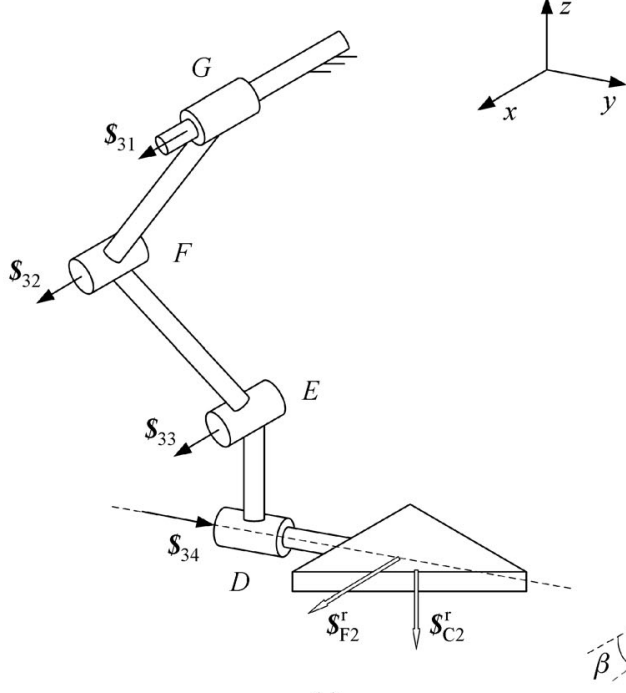

(a)

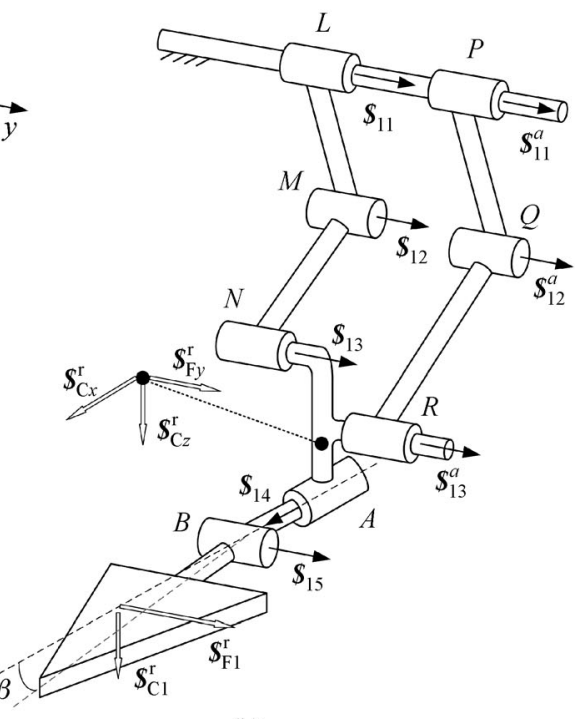

(b)

Fig. 6 Constraints analysis of (a) limb 3, (b) limb 1 and the configuration that the platform rotates about $v$ first.

where $\boldsymbol{S}_{\mathrm{C} x}^{\mathrm{r}}, \boldsymbol{S}_{\mathrm{F} y}^{\mathrm{r}}$, and $\boldsymbol{S}_{\mathrm{C} z}^{\mathrm{r}}$ are the wrenches acted upon the parallel part of limb associated with joint $A$, and meanwhile they are overconstraints due to the identical limb structure of the two sub-chains in $L M N-P Q R$. As a result, the standard base of output twist system of the parallel part is

$$
\left\{\begin{array}{l}
\boldsymbol{S}_{\mathrm{p} 1}=\left(\begin{array}{llllll}
0 & 0 & 0 ; & 1 & 0 & 0
\end{array}\right), \\
\boldsymbol{S}_{\mathrm{p} 2}=\left(\begin{array}{llllll}
0 & 0 & 0 ; & 0 & 0 & 1
\end{array}\right), \\
\boldsymbol{S}_{\mathrm{p} 3}=\left(\begin{array}{llllll}
0 & 1 & 0 ; & 0 & 0 & 0
\end{array}\right),
\end{array}\right.
$$

where the subscript $\mathrm{p}$ denotes the parallel part. After connecting the series part, the twist system of limb 1 can be expressed as

$$
\left\{\begin{array}{l}
\boldsymbol{S}_{\mathrm{p} 1}=\left(\begin{array}{llllll}
0 & 0 & 0 ; & 1 & 0 & 0
\end{array}\right), \\
\boldsymbol{S}_{\mathrm{p} 2}=\left(\begin{array}{llllll}
0 & 0 & 0 ; & 0 & 0 & 1
\end{array}\right), \\
\boldsymbol{S}_{\mathrm{p} 3}=\left(\begin{array}{llllll}
0 & 1 & 0 ; & 0 & 0 & 0
\end{array}\right), \\
\boldsymbol{S}_{14}=\left(\begin{array}{llllll}
1 & 0 & 0 ; & 0 & a_{4} & b_{4}
\end{array}\right), \\
\boldsymbol{S}_{15}=\left(\begin{array}{llllll}
0 & 1 & 0 ; & a_{5} & 0 & b_{5}
\end{array}\right) .
\end{array}\right.
$$

The standard base of the wrench system of limb 1 still is

$$
\left\{\begin{array}{l}
\boldsymbol{S}_{\mathrm{C} 1}^{\mathrm{r}}=\left(\begin{array}{llllll}
0 & 0 & 0 ; & 0 & 0 & 1
\end{array}\right), \\
\boldsymbol{S}_{\mathrm{F} 1}^{\mathrm{r}}=\left(\begin{array}{llllll}
0 & 1 & 0 ; & 0 & 0 & 0
\end{array}\right) .
\end{array}\right.
$$

When the platform rotates a certain angle about the axis parallel to $y$-axis ( $v$ ) first, the wrench system of the platform will not be changed, in which $\boldsymbol{S}_{\mathrm{C} 1}^{\mathrm{r}}$ is perpendicular to the plane determined by joints $A$ and $B$, and $\boldsymbol{S}_{\mathrm{F} 1}^{\mathrm{r}}$ is located in this plane and perpendicular and intersect with the axis of joint $A$. At this configuration, there are one common constraint formed by $\boldsymbol{S}_{\mathrm{C} 1}^{\mathrm{r}}, \boldsymbol{S}_{\mathrm{C} 2}^{\mathrm{r}}, \boldsymbol{S}_{\mathrm{C} 3}^{\mathrm{r}}$, and $\boldsymbol{S}_{\mathrm{C} z}^{\mathrm{r}}$, i.e., $\lambda=1$ and three overconstraints containing $\boldsymbol{S}_{\mathrm{C} x}^{\mathrm{r}}, \boldsymbol{S}_{\mathrm{F} y}^{\mathrm{r}}$, and $\boldsymbol{S}_{\mathrm{F} 3}^{\mathrm{r}}$, i.e., $v=3$. Thus, the output DOF of the platform and the DOF of the mechanism are

$$
\begin{gathered}
F=6-C=6-3=3, \\
M=d(n-g-1)+\sum_{i=1}^{g} f_{i}+v \\
=5 \times(14-16-1)+16+3=4 .
\end{gathered}
$$

From Eqs. (20) and (21), it can be drawn that a passive DOF still exist when the platform rotates about axis parallel to $y$ first, but it is controllable due to four actuators are installed in the base. In this case, the mechanism is kinematic redundant.

However, when the platform rotates about the axis parallel to $x$-axis $(\boldsymbol{u})$ first, as shown in Fig. 7, the twist associated with joint $B$ become

$$
\boldsymbol{\$}_{15}=\left(\begin{array}{llllll}
0 & m & n ; a_{5} & b_{5} & c_{5}
\end{array}\right) .
$$

The standard base of the wrench system of limb 1 become

$$
\boldsymbol{S}_{\mathrm{F} 1}^{\mathrm{r}}=\left(\begin{array}{cccccc}
0 & 1 & 0 ; & -a_{4} & 0 & -\frac{b_{5}}{n}
\end{array}\right) .
$$

Hence, this limb becomes an $\mathrm{L}_{\mathrm{F}}$ in the configuration of Fig. 7. There are three independent constraints for the platform i.e., $C=3$, and five overcontraints for the whole PM, i.e., $v=5$.

$$
F=6-C=6-3=3,
$$




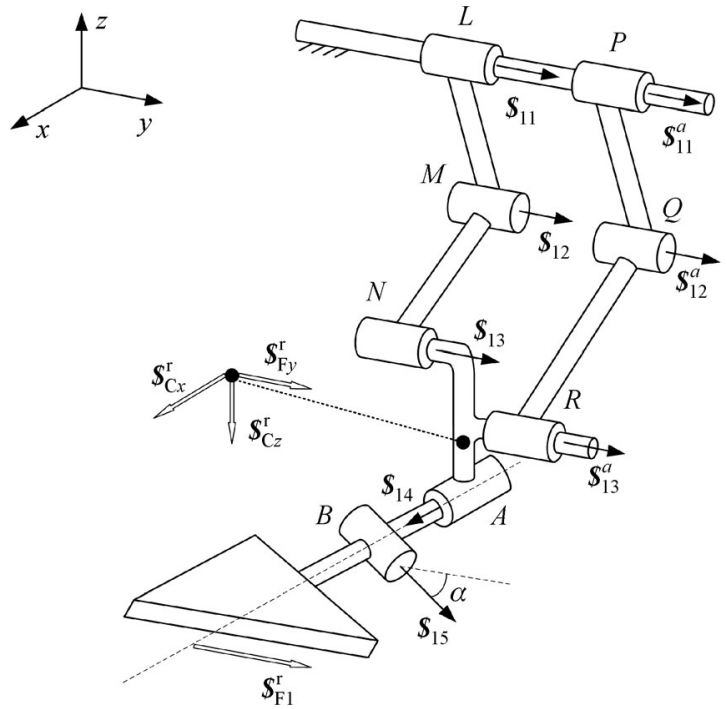

Fig. 7 The limb 1 and the configuration that the platform rotates about $\boldsymbol{u}$ first.

$$
\begin{aligned}
M & =d(n-g-1)+\sum_{i=1}^{g} f_{i}+v \\
& =6 \times(14-16-1)+16+5=3 .
\end{aligned}
$$

It can be seen that the passive DOF is eliminated by the interference of limbs and the rotation about axis parallel to $y$ is interfered, but the rotation about the movable axis $v$ is remained. Compared to the situation of rotating about $v$ first, the nature of the rotational DOF has changed when rotating about $\boldsymbol{u}$ first. In this case, the mechanism is actuated redundant.

\section{A class of 5-axis collaborative manipulators}

As the 2R1T (rotations about $x$ and $y$ directions, translation along $z$ direction) spindle heads are obtained, the following work is to design a bottom collaborative platform with $2 \mathrm{D}$ translations along $x$ and $y$ directions to construct the 5-axis collaborative manipulators. In order to improve rigidity and stability, a superimposed series-platform is built. This $2 \mathrm{~T}$ bottom series-platform is actuated by two motors and realizes motion transmission depending on the screw mandrels and sliding rods connected in the base and the screw mandrels, as illustrated in Fig. 8.

Then, a class of 5-axis collaborative manipulators can be obtained by connecting the 2R1T spindle heads and the $2 \mathrm{~T}$ series-platform, as shown in Fig. 9. For these collaborative manipulators, the basic mobility performance is identical due to the theoretically equivalent limb structures of the $2 \mathrm{R} 1 \mathrm{~T}$ spindle heads. These manipulators are represented as $4 \dot{\mathrm{R}} \dot{\mathrm{R}} \dot{\mathrm{R}}-[\mathrm{PLA}]-[\dot{\mathrm{P}} \ddot{\mathrm{P}}]$,
4Pنं $\dot{\mathrm{R}}-[\mathrm{PLA}]-[\dot{\mathrm{P}} \ddot{\mathrm{P}}], 4 \dot{\mathrm{R}} \mathrm{P} \dot{\mathrm{R}}-[\mathrm{PLA}]-[\dot{\mathrm{P}} \ddot{\mathrm{P}}]$, respectively, in which $[\ddot{P} \ddot{P}]$ denotes the bottom $2 \mathrm{~T}$ series-platform and the dots above $\mathrm{P}$ are used to mark the different directions of the prismatic pairs.

\section{The rotational capability illustration example}

Since the proposed 5-axis collaborative manipulators share the same [PLA], and have the limbs with the same motion function, the rotational capability of these manipulators is almost the same. This section takes the $4 \mathrm{PR} \dot{\mathrm{R}}-[\mathrm{PLA}]-[\dot{\mathrm{P}} \ddot{\mathrm{P}}]$ $\mathrm{PM}$ as an example to study the rotational capability because the prismatic actuators make the kinematics easy to solve and calculate compared with the revolute counterpart. Based on the above design process, the orientation of joint $A$ by using kinematic redundant limb is the key factor to achieve 2D high rotational DOF. As a result, this section analyses the analytical kinematic conditions for orienting joint $A$. In order to know the reason of the $2 \mathrm{D}$ high rotational capability, this section also investigates the rotation decoupling properties of the example PM.

\subsection{Inverse kinematics}

The geometrical model of the 4PRं $\dot{R}$-[PLA] PM is depicted in Fig. 10. The global reference frame $O_{\mathrm{b}}-X_{\mathrm{b}}(\boldsymbol{i}) Y_{\mathrm{b}}(\boldsymbol{j}) Z_{\mathrm{b}}(\boldsymbol{k})$ is attached at the center of the manipulator with $Z_{\mathrm{b}}(\boldsymbol{k})$-axis normal to the base plate and $X_{\mathrm{b}}(\boldsymbol{i})$-axis parallel with $O_{1} P_{1}$. The moving reference frame $O-x y z$ locates at the center of the moving platform. The $y$-axis coincides with the rotating axis of joints $C$ and $D$ as well as directs to joint $D$, and $z$-axis is perpendicular to the moving platform. The dimension parameters are denoted in Table 1.

The objective of the inverse kinematics solution is to define a mapping from the position and posture of the moving platform to the set of inputs. For the 4PRंR -[PLA] parallel spindle head, it can be described as: Given the position ${ }^{\mathrm{b}} O=\left(\begin{array}{lll}x & y & z\end{array}\right)^{\mathrm{T}}$ of point $O$ that related to the base frame and orientation $\alpha$ about $x$-axis of moving reference and $\beta$ about $y$-axis of moving reference, compute the linear displacements $\left(q_{1}, q_{2}, q_{3}, q_{4}\right)$ of the actuators. In order to be convenient for computation, the moving platform is artificially assumed to rotate about $y$-axis by $\beta$ first and then to rotate about $x$-axis by $\alpha$, the rotational matrix represented by $\left[R^{\beta \alpha}\right]$ can be calculated as

$$
\begin{aligned}
{\left[R^{\beta \alpha}\right] } & =\left[R^{\beta}\right]\left[R^{\alpha}\right] \\
& =\left(\begin{array}{ccc}
\cos \beta & \sin \alpha \sin \beta & \cos \alpha \sin \beta \\
0 & \cos \alpha & -\sin \alpha \\
-\sin \beta & \cos \beta \sin \alpha & \cos \alpha \cos \beta
\end{array}\right) .
\end{aligned}
$$




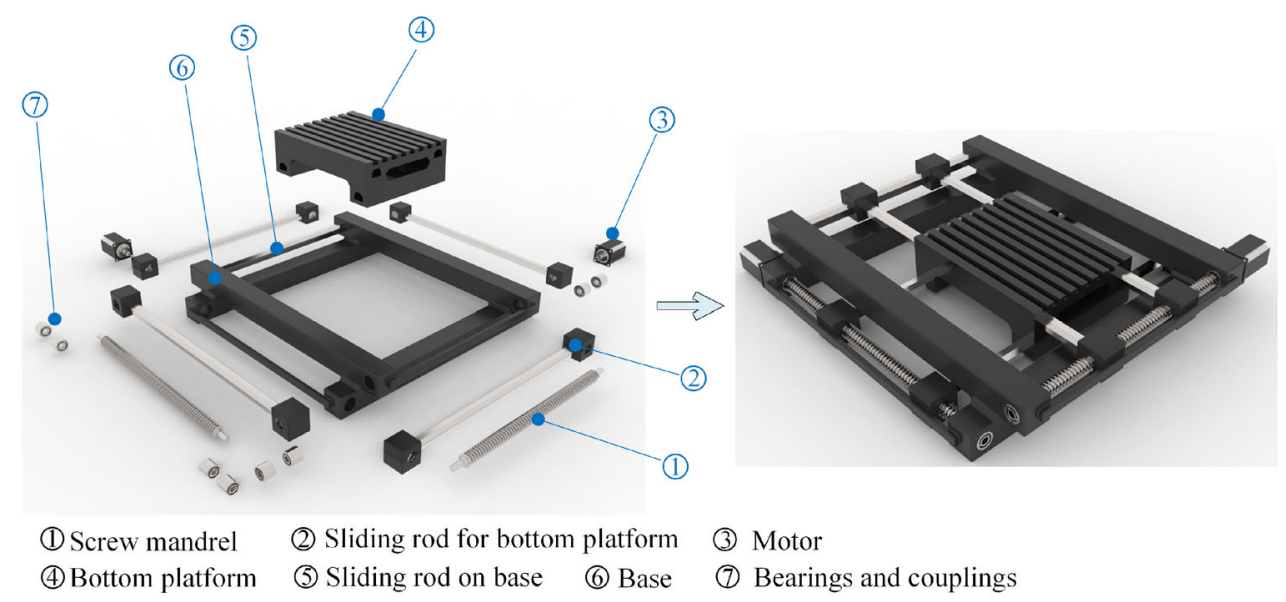

Fig. 8 The part lists and exploded pictures and the assembly drawing of the bottom collaborative platform.

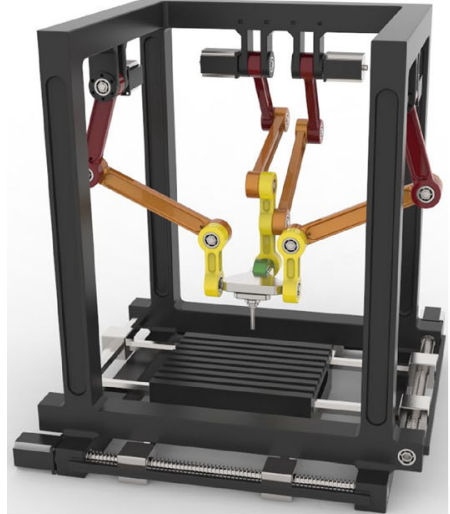

(a)

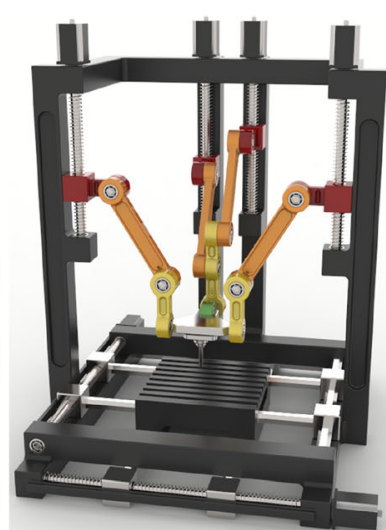

(b)

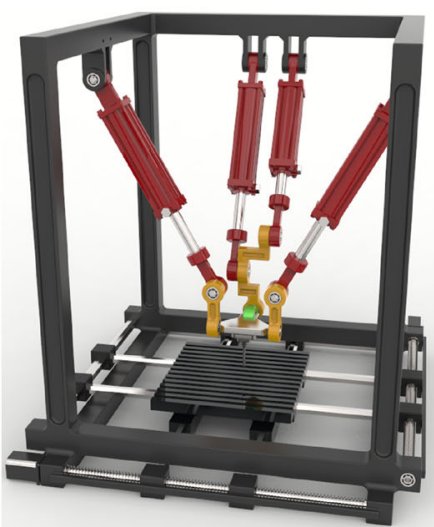

(c)

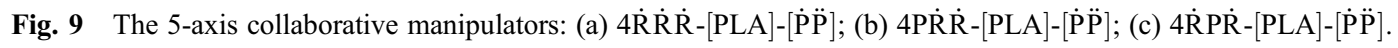

Table 1 Architecture parameters of 4PRंR -[PLA] head

\begin{tabular}{lc||lc}
\hline Parameter & Value & Parameter & Value \\
\hline$l_{O B}, l_{O C}, l_{O D}$ & $60 \mathrm{~mm}$ & $l_{A Q_{1}}^{y}$ & $20 \mathrm{~mm}$ \\
$l_{A B}$ & $30 \mathrm{~mm}$ & $l_{A Q_{1}}^{z}$ & $30 \mathrm{~mm}$ \\
$l_{Q_{2} C}, l_{Q_{3} C}$ & $30 \mathrm{~mm}$ & $P_{1}^{b}$ & $\left(-100,-20, q_{1}\right)$ \\
$l_{A Q_{4}}^{v}$ & $20 \mathrm{~mm}$ & $P_{2}^{b}$ & $\left(0,-100, q_{2}\right)$ \\
$l_{A Q_{4}}^{z}$ & $15 \mathrm{~mm}$ & $P_{3}^{b}$ & $\left(0,100, q_{3}\right)$ \\
$l_{P_{i} Q_{i}}$ & $150 \mathrm{~mm}$ & $P_{4}^{b}$ & $\left(-100,20, q_{4}\right)$ \\
\hline
\end{tabular}

The coordinates of joints $B, C$, and $D$ represented in the moving reference are ${ }^{\mathrm{p}} B=\left(\begin{array}{lll}-l_{O B} & 0 & 0\end{array}\right)^{\mathrm{T}}$, ${ }^{\mathrm{p}} C=\left(\begin{array}{lll}0 & -l_{O C} & 0\end{array}\right)^{\mathrm{T}}$, and ${ }^{\mathrm{p}} D=\left(\begin{array}{lll}0 & l_{O D} & 0\end{array}\right)^{\mathrm{T}}$, where $l_{O B}, l_{O C}$, and $l_{O D}$ denote the distance between $O$ and $B, C$, $D$, respectively. As shown in Fig. 10(b), using $l_{Q_{2} C}$ and $l_{Q_{3} D}$ represent the length of link $Q_{2} C$ and $Q_{3} D$, respectively, the coordinates of $Q_{2}$ and $Q_{3}$ expressed in the moving reference are

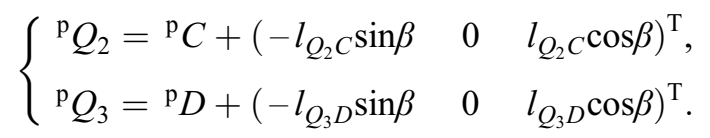

Thus, the coordinates of $Q_{2}$ and $Q_{3}$ represented in the base reference can be calculated as

$$
{ }^{\mathrm{b}} Q_{i}={ }^{\mathrm{b}} O+\left[R^{\beta \alpha}\right] \cdot{ }^{\mathrm{p}} Q_{i}, i=2 \text { and } 3 .
$$

Joint $B$ represented in the base frame is

$$
{ }^{\mathrm{b}} B={ }^{\mathrm{b}} O+\left[R^{\beta \alpha}\right] \cdot{ }^{\mathrm{p}} B .
$$

Since the function of the redundant limb (limb 4) is to maintain the parallel relationship between link $A B$ and the horizontal plane, the geometric structure of joint $A$ relative to the base reference frame satisfies Fig. 10(c). As a result, the coordinate of joint $A$ relative to the base is

$$
{ }^{\mathrm{b}} A={ }^{\mathrm{b}} B+\left(\begin{array}{lll}
-l_{A B} & 0 & 0
\end{array}\right)^{\mathrm{T}},
$$

where $l_{A B}$ denotes the length of link $A B$. Links $A Q_{1}$ and $A Q_{4}$ are always parallel to the $j k$-plane when link $A B$ keeps 
parallel with the horizontal plane. Thus, the position of joints $Q_{1}$ and $Q_{4}$ relative to the base reference frame are

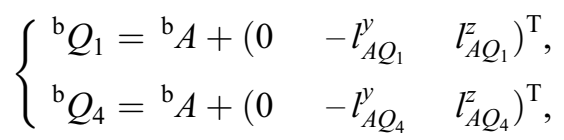

where $l_{A Q_{i}}^{y}$ and $l_{A Q_{i}}^{z}$ represent the $y$ and $z$ component of the length of link $A Q_{i}(i=1$ and 4$)$, respectively. Depending on the geometrical constraints, the following equality can be satisfied

$$
\left\|{ }^{\mathrm{b}} P_{i}-{ }^{\mathrm{b}} Q_{i}\right\|=l_{P_{i} Q_{i}}, i=1,2,3,4,
$$

where $l_{P_{i} Q_{i}}$ denotes the length of link $P_{i} Q_{i}$. The above relationship leads to

$$
M_{i} q_{i}^{2}+N_{i} q_{i}+T_{i}=0
$$

where $M_{i}, N_{i}$, and $T_{i}$ are associated with parameters $\left(\begin{array}{lll}z & \alpha & \beta\end{array}\right)$ mentioned above.

Further, Eq. (33) result in

$$
q_{i}=\frac{-N_{i} \pm \sqrt{N_{i}^{2}-4 M_{i} T_{i}}}{2 M_{i}} .
$$

Hence, the required actuator inputs of $q_{1}, q_{2}, q_{3}$ and $q_{4}$ can be computed from Eq. (34) if the values of the position and orientation of the platform are given.

\subsection{Analytical kinematic conditions for orienting joint $A$}

Figures 11(a)-11(c) show the geometrical modes of limb 1 of the $4 \dot{R} \dot{R} \dot{R}-[P L A]$ head, 4 P $\dot{R} \dot{R}$-[PLA] head and $4 \dot{R} P \dot{R}$ [PLA] head, respectively. If joint $A$ is parallel with $X_{\mathrm{b}}$-axis constantly, its position is determined by the position of $O$ and the rotational angle $\beta$. In $X_{\mathrm{b}} Z_{\mathrm{b}}$-plane, position of point
$O$ can be represented as ${ }^{b} O=(0, z)^{\mathrm{T}}$, Then position of $B, A$, $Q_{1}$ and $Q_{4}$ can be calculated out:

$$
\begin{gathered}
{ }^{\mathrm{b}} B={ }^{\mathrm{b}} O+\left(\begin{array}{cc}
-l_{O B} \cos \beta & l_{O B} \sin \beta
\end{array}\right)^{\mathrm{T}}, \\
{ }^{\mathrm{b}} A={ }^{\mathrm{b}} B+\left(\begin{array}{ll}
-l_{A B} & 0
\end{array}\right)^{\mathrm{T}}, \\
{ }^{\mathrm{b}} Q_{i}={ }^{\mathrm{b}} A+\left(\begin{array}{ll}
0 & l_{A Q_{i}}
\end{array}\right)^{\mathrm{T}}, i=1 \text { and } 4 .
\end{gathered}
$$

Position of $P_{i}$ corresponding to the $X_{\mathrm{b}} Z_{\mathrm{b}}$-plane in the $4 \dot{\mathrm{R}} \dot{\mathrm{R}} \dot{\mathrm{R}}-[\mathrm{PLA}]$ head is ${ }^{\mathrm{b}} P_{i}=\left(-l_{O_{i} O_{b}}-l_{O_{i} P_{i}} \sin \beta\right.$ $\left.-l_{O_{i} P_{i}} \cos \beta\right)^{\mathrm{T}}$ position of $P_{i}$ corresponding to the $X_{\mathrm{b}} Z_{\mathrm{b}}$ plane in the 4Pنं $\dot{\mathrm{R}}$-[PLA] head is known in Section 5.1, and position of $O_{i}$ corresponding to the $X_{\mathrm{b}} Z_{\mathrm{b}}$-plane in $4 \dot{\mathrm{R} P \dot{R}}-[\mathrm{PLA}]$ head can be represented as ${ }^{\mathrm{b}} O_{i}=\left(-l_{O_{i} O_{b}} 0\right)^{\mathrm{T}}$. For the $4 \dot{\mathrm{R}} \dot{\mathrm{R}} \dot{\mathrm{R}}-[\mathrm{PLA}]$ head and 4PRं -[PLA] head, the analytical kinematic conditions when joint $A$ is oriented can be solved by

$$
\left\|{ }^{\mathrm{b}} P_{i}-{ }^{\mathrm{b}} Q_{i}\right\|=l_{P_{i} Q_{i}}, i=1 \text { and } 4 .
$$

For the $4 \dot{R} P \dot{R}-[P L A]$ head, the analytical kinematic conditions when joint $A$ is oriented can be obtained by

$$
q_{i}=\left\|{ }^{\mathrm{b}} O_{i}-{ }^{\mathrm{b}} Q_{i}\right\|, i=1 \text { and } 4 .
$$

When the input parameters $q_{1}$ and $q_{4}$ satisfy the results solved by Eqs. (38) and (39), joints $A$ in this type of PMs can be guaranteed to be oriented parallel to $X_{\mathrm{b}}$-axis.

According to the results solved by Eqs. (38) and (39), the relationship between the input parameters $q_{1}$ and $q_{4}$ to keep joint $A$ horizontal can be plotted out as Fig. 12 when the architecture parameters are given as Table 2. Concretely, Fig. 12 visualizes the variation of input values of the two actuators $q_{1}$ and $q_{4}$ with position and posture of
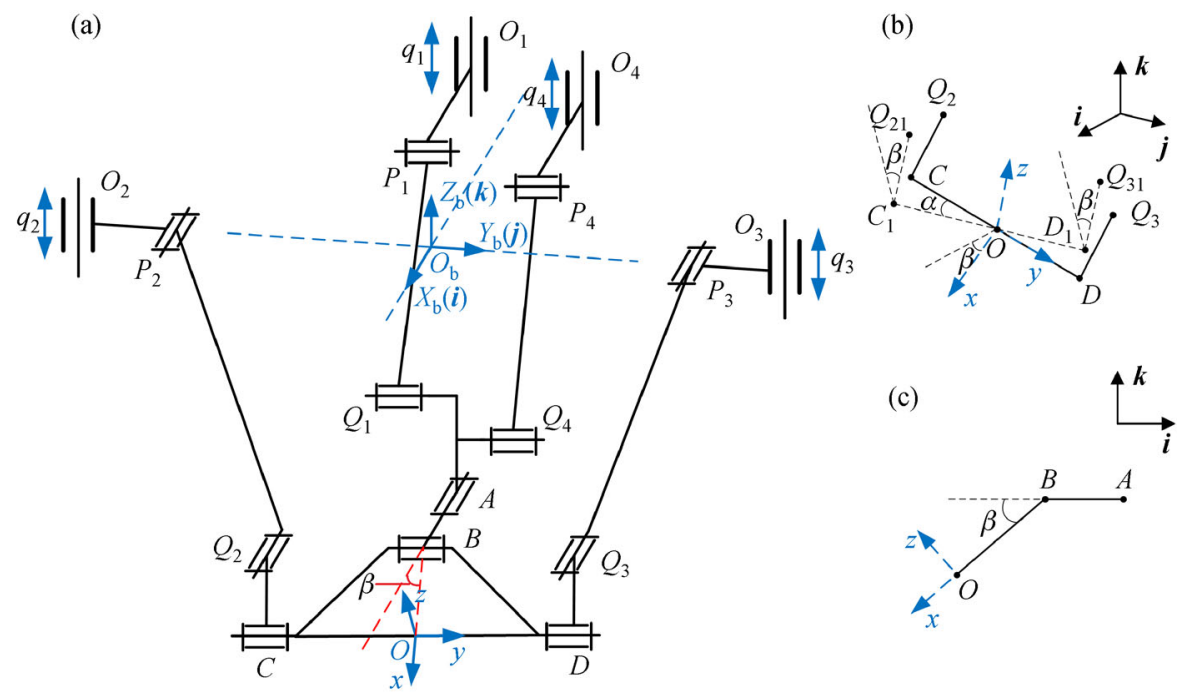

Fig. 10 Geometrical model of the 4PRंR -[PLA] spindle head. (a) The whole structure; (b) joins $Q_{2}$ and $Q_{3}$; (c) joins $A$. 


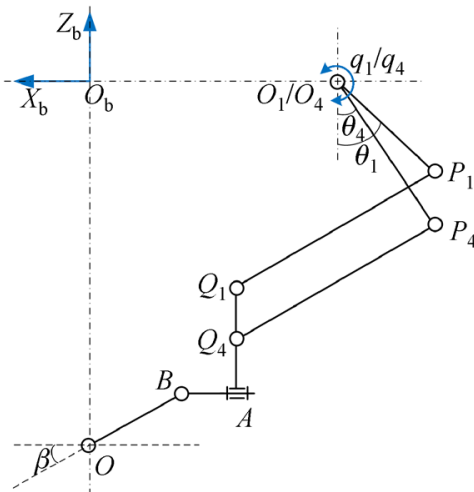

(a)

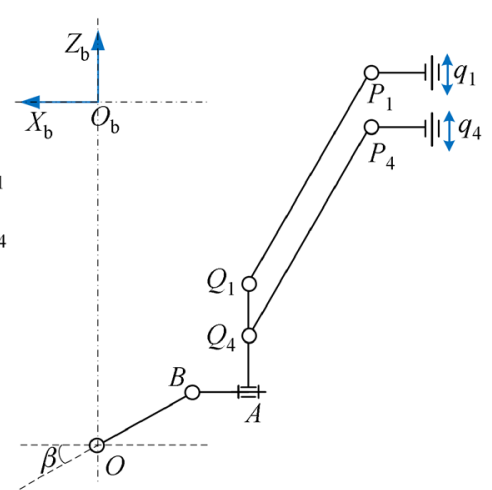

(b)

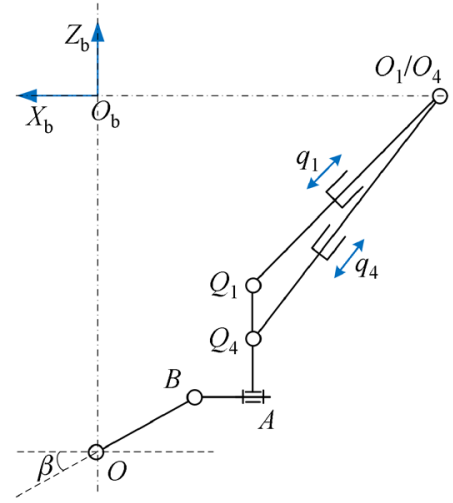

(c)

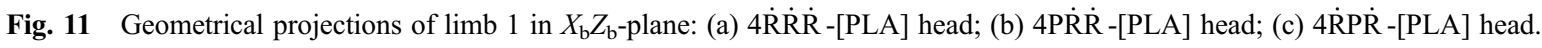

the moving platform, when the requirement of orienting joint $A$ is satisfied. If the input values of $q_{1}$ and $q_{4}$ follow the analytical kinematic conditions solved by Eqs. (38) and (39) and obey the corresponding magnitudes at a certain position and posture $(z, \beta)$ in Fig. 12 , the passive DOF in limb 1 can be determined and controlled to maintain joint $A$ parallel to $X_{\mathrm{b}}$-axis when the platform rotates about $y$-direction first.

\subsection{The 1D orientation workspace}

According to the inverse kinematics, the $1 \mathrm{D}$ rotational capability can be investigated after setting $\alpha$ and $\beta$ equal to $0^{\circ}$, respectively. When giving the actuators' threshold of $q_{i} \in[-100 \mathrm{~mm}, 200 \mathrm{~mm}]$ and limiting the motion ranges of $\alpha, \beta$, and $Z$ to $\left[-180^{\circ}, 180^{\circ}\right],\left[-180^{\circ}, 180^{\circ}\right]$ and $[-300 \mathrm{~mm}, 0 \mathrm{~mm}]$, the relationships between the rotational angles about $x$-direction and the rotational angles about $y$-direction with the displacement along $z$-direction can be drawn in Fig. 13, respectively. Considering the minimal distance and interference among linkages as well as singularity, we artificially give two stringent constraints for rotational angles during generating the workspace: Position of joint $A$ does not exceed $Y_{\mathrm{b}} Z_{\mathrm{b}}$-plane and the sum of rotational angles is not greater than $180^{\circ}$. These two constraints are able to guarantee no interference between limbs and can be mathematically represented as

$$
\left\{\begin{array}{l}
X_{A} \leqslant 0, \\
\alpha+\beta \leqslant 180^{\circ} .
\end{array}\right.
$$

Some conclusions can be obtained based on the 1D orientation workspaces:

- The 1D rotation angles $\alpha$ and $\beta$ decrease with the

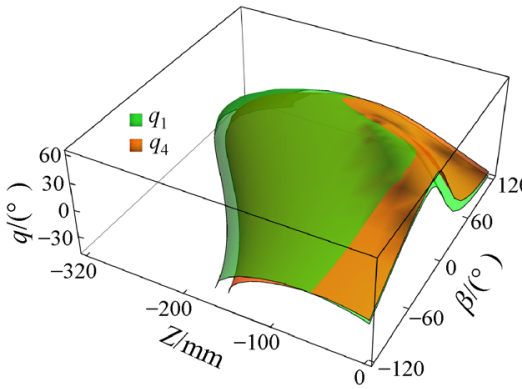

(a)

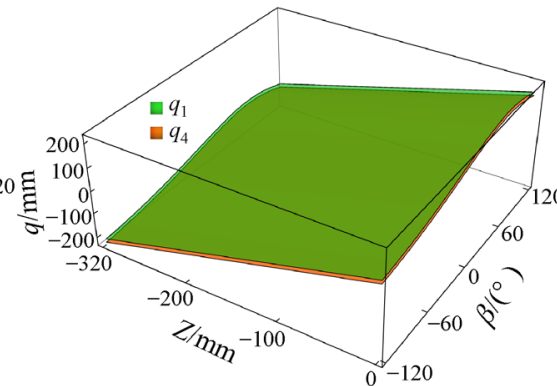

(b)

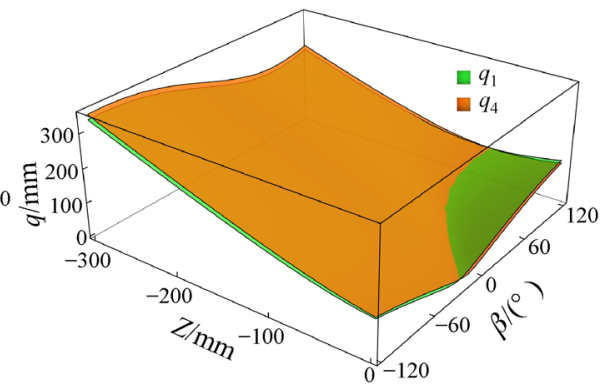

(c)

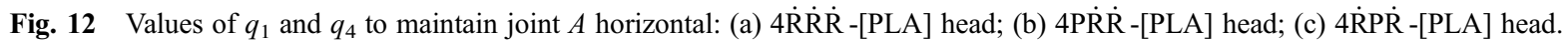

Table 2 Architecture parameters of limb 1 in $X_{\mathrm{b}} Z_{\mathrm{b}}$-plane

\begin{tabular}{|c|c|c|c|c|c|c|c|}
\hline Spindle head & $l_{O B} / \mathrm{mm}$ & $l_{A B} / \mathrm{mm}$ & $l_{A Q_{1}} / \mathrm{mm}$ & $l_{A Q_{4}} / \mathrm{mm}$ & $l_{P_{i} Q_{i}} / \mathrm{mm}$ & $l_{O_{i} P_{i}} / \mathrm{mm}$ & $l_{O_{i} O_{b}} / \mathrm{mm}$ \\
\hline $4 \dot{\mathrm{R}} \dot{\mathrm{R}} \dot{\mathrm{R}}-[\mathrm{PLA}]$ & 60 & 30 & 30 & 15 & 150 & 90 & 150 \\
\hline 4PR் $\dot{R}$-[PLA] & 60 & 30 & 30 & 15 & 150 & - & - \\
\hline $4 \dot{\mathrm{R} P \dot{R}}-[\mathrm{PLA}]$ & 60 & 30 & 30 & 15 & - & - & 100 \\
\hline
\end{tabular}


decreasing of the displacement along $z$-axis.

- The maximal rotational angles about both $x$-direction and $y$-direction are larger than $\pm 90^{\circ}$ before optimization, which are much better than the $\mathrm{Z} 3$ and $\mathrm{A} 3$ spindle heads whose $2 \mathrm{D}$ rotational angles are limited to about $\pm 50^{\circ}[34$ 36].

- The angle range is symmetric distribution in the positive and negative intervals for the rotation about $x$-direction but not for the rotation about $y$-direction because the structure is symmetrical in the $X_{\mathrm{b}} Z_{\mathrm{b}}$-plane but asymmetrical in the $Y_{\mathrm{b}} Z_{\mathrm{b}}$-plane.

- In Fig. 13(b), the overall distribution of the orientation workspace about $y$-axis is asymmetric. When the moving platform approaches the upper limit position, the actuators on limb 1 are also close to the upper limit position. At this time, the moving platform can no longer rotate in the positive direction because the rotation in the positive direction depends on the upward displacement of the actuators on limbs. That is why there is a blank area in the positive direction of rotation about $y$-axis near the $z$-direction translation value of 0 . By the same reason, there is a blank area in the negative direction of rotation about $y$-axis when the moving platform nears the lower limit position. When the moving platform is far from the limit position, i.e., the value of $z$ is about -190 to $-30 \mathrm{~mm}, \beta$ is symmetrical about $0^{\circ}$.

Figure 14 illustrates the example rotational configurations. The example 1D rotational configurations are illustrated in Figs. 14(a), 14(b), 14(d), and 14(e), where Figs. 14(a) and 14(d) demonstrate the large rotational angles about $x$-direction by $\pm 90^{\circ}$, and Figs. 14(b) and 14(e) show the large rotational angles about $y$-direction by $\pm 90^{\circ}$.

\subsection{The 2D orientation workspace at constant-position}

There is usually some interference between the multidimension rotations, i.e., if the platform rotates about a certain direction first, the rotational range about other

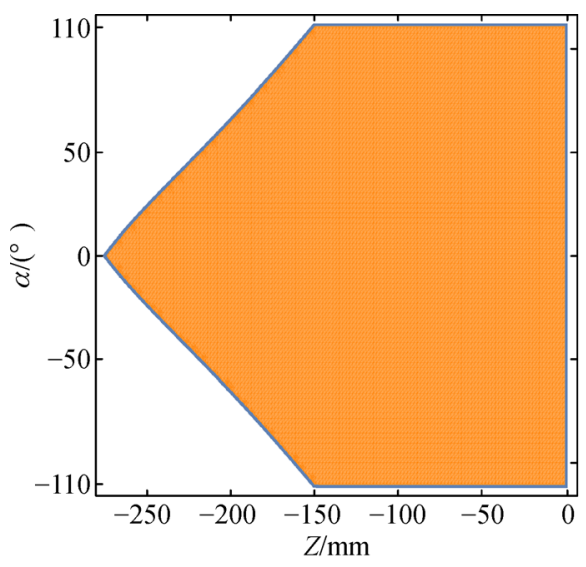

(a) directions will decrease because of the diminution of the corresponding translational workspace. In order to investigate the interference between the rotations and the 2D rotational capability, the $2 \mathrm{D}$ orientation workspace at different locations are mapped as shown in Fig. 15. Since the translational workspace along $z$-direction is between -300 and $0 \mathrm{~mm}$ based on the architecture parameters, four locations that $z=-240, z=-180, z=-120$, and $z=-60 \mathrm{~mm}$ are selected to learn about the 2D workspace. Figures 14(c) and 14(f) show the example 2D rotational configurations by $\pm 45^{\circ}$. There are two conclusions based on the $2 \mathrm{D}$ orientation workspace:

- All the 2D rotational workspace at the example locations are closed and continuous mapping surface, i.e., there is no blank inside the workspace boundary, which indicates there is no rotational interference as well as the orientation workspace singularity. Concretely, if the platform rotates about one direction by a certain angle, it can move to the desired angle about another direction without obstacles within the orientation workspace.

- The 2D maximal rotational angles increase with the increase of the displacement along $z$-direction.

It should be mentioned that value $\beta$ is asymmetric in Fig. 15(a) but symmetric in Figs. 15(b)-15(d). That is because when $z=-240 \mathrm{~mm}$, according to Fig. 13(b), the rotation capability in negative direction about $y$-axis becomes worse. However, the reachable rotational angles are symmetrical when $z=-180, z=-120$, and $z=-60 \mathrm{~mm}$ as illustrated in Fig. 13(b).

\subsection{Rotation decoupling properties}

In order to reveal the reason that makes the moving platform have 2D large rotational angles, this part investigates the relationships between the rotations and the input parameters. When letting $z=-120 \mathrm{~mm}$ and $\beta=$ $0^{\circ}$, the relationships between rotation angle $\alpha$ about $x$-axis and the four input parameters $q_{1}, q_{2}, q_{3}$, and $q_{4}$ can be obtained as Fig. 16(a). It shows that when the moving

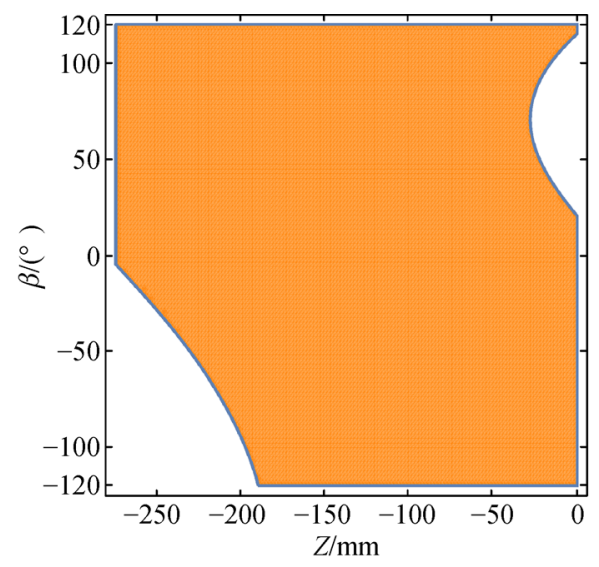

(b)

Fig. $131 \mathrm{D}$ rotation capabilities along $z$-axis: (a) $\beta=0^{\circ}$; (b) $\alpha=0^{\circ}$. 


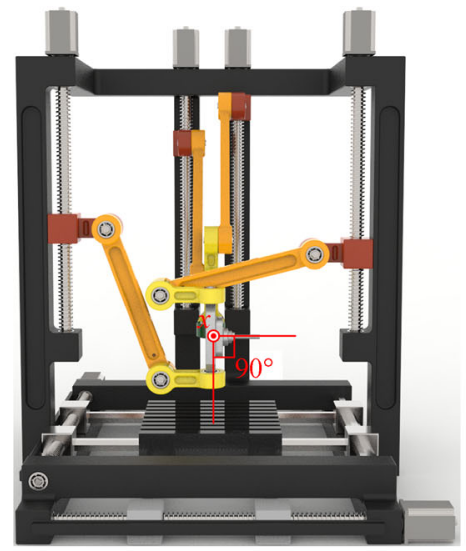

(a)

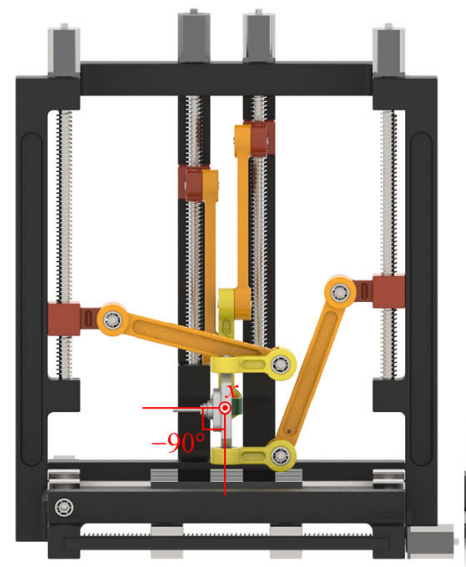

(d)

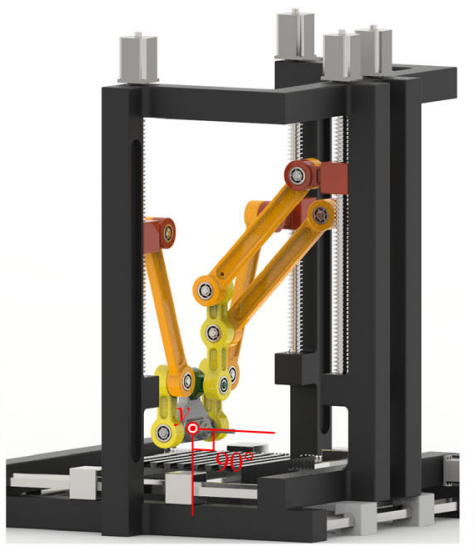

(b)

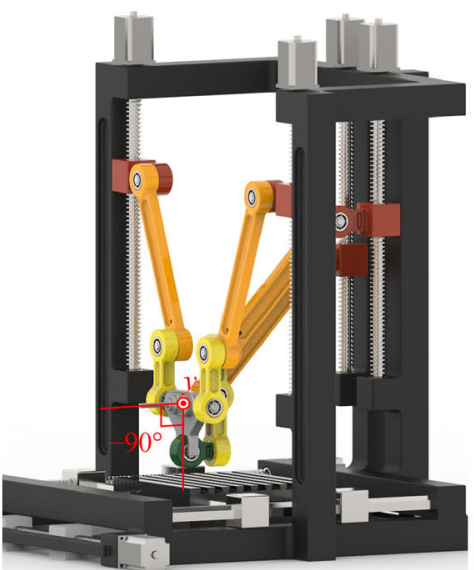

(e)

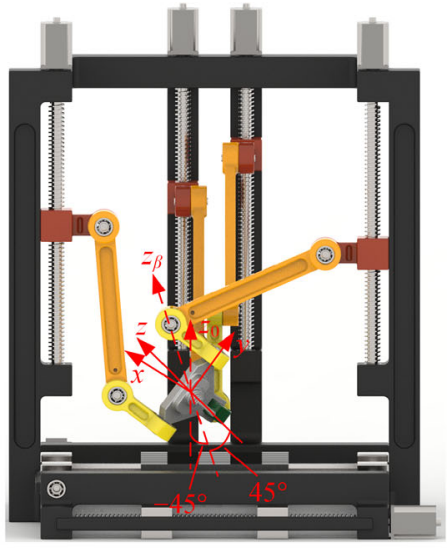

(c)

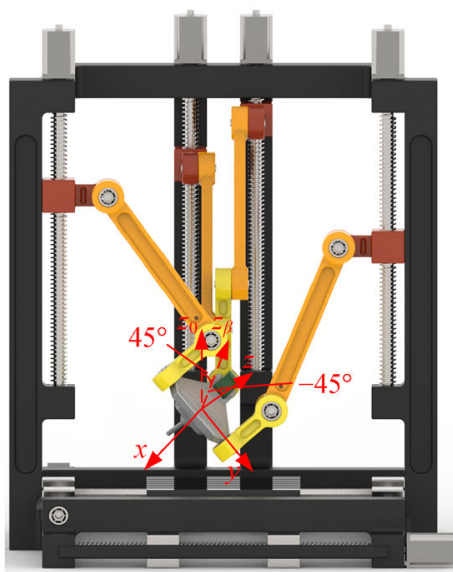

(f)

Fig. 14 The example rotational configurations about (a) $x$-direction by $90^{\circ}$, (b) $y$-direction by $90^{\circ}$, (c) $x$-direction by $45^{\circ}$ and $y$-direction by $-45^{\circ}$, (d) $x$-direction by $-90^{\circ}$, (e) $y$-direction by $-90^{\circ}$, and (f) $x$-direction by $-45^{\circ}$ and $y$-direction by $45^{\circ}$.

platform is rotating about $x$-axis, the input parameters $q_{1}$ and $q_{4}$ remain unchanged and $q_{2}$ and $q_{3}$ vary with $\alpha$, i.e., the actuators in limb 1 keep still, and only the actuators in limbs 2 and 3 drive the rotation about $x$-axis. It indicates that the rotation about $x$-axis is partially decoupled. Similarly, when letting $z=-120 \mathrm{~mm}$ and $\alpha=0^{\circ}$, the relationships between rotation angle $\beta$ about $y$-axis and the four input parameters $q_{1}, q_{2}, q_{3}$, and $q_{4}$ can be figured out as Fig. 16(b). Conversely, the input parameters $q_{2}$ and $q_{3}$ remain unchanged with same value and $q_{1}$ and $q_{4}$ vary with $\beta$, i.e., the actuators in limbs 2 and 3 keep still, and only the actuators in limb 1 drive the rotation about $y$-axis. It illustrates that the rotation about $y$-axis is also partially decoupled.

The partially decoupled rotation, which means the actuators drive each rotation DOF relatively independent and generate as little mutual interference as possible between rotations, always brings large orientation workspace for the PMs. Here, it can be concluded that the design of [PLA] with 2D rotational axes as well as the synthesis of the kinematic redundant limbs structure make the mechanisms have partially decoupled rotation perfor- mance, which is able to bring 2D large rotational angles.

\section{Conclusions}

This paper presents a class of 5-axis collaborative manipulators with 2D large rotational angles. Design of the platform structure with $2 \mathrm{D}$ rotational axes is an important step of the synthesis procedure, which is the base of the limb structural synthesis. The constraint screw theory, which reveals the relationship between constraints and the motions of the PMs, is used to synthesize the limbs structures connected in the platform. The redundant limb guarantees the $2 \mathrm{D}$ high rotational capability by providing partially decoupled rotations through orienting the rotational axis. The redundant $2 \mathrm{R} 1 \mathrm{~T}$ parallel spindle heads are proved to have $2 \mathrm{D}$ large rotational angles more than $\pm 90^{\circ}$. The proposed 2R1T heads are better than the famous Z3 and $\mathrm{A} 3 \mathrm{~h}$ head in terms of the rotational angles. The obtained 5 -axis collaborative manipulators can be potentially used in the industrial applications such as the machine tools and the $3 \mathrm{D}$ printers. The following researches will focus on the 


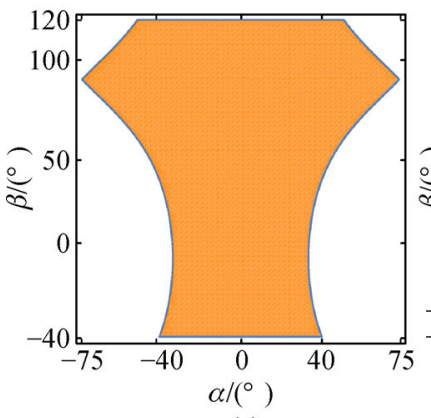

(a)

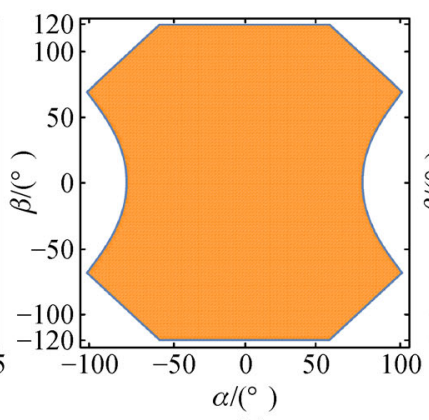

(b)

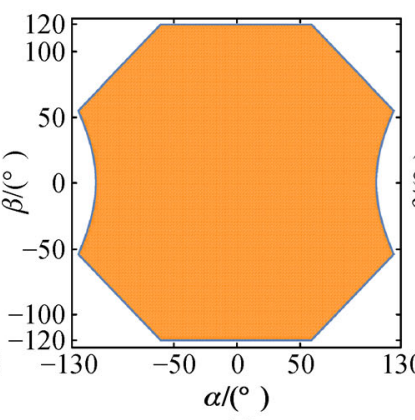

(c)

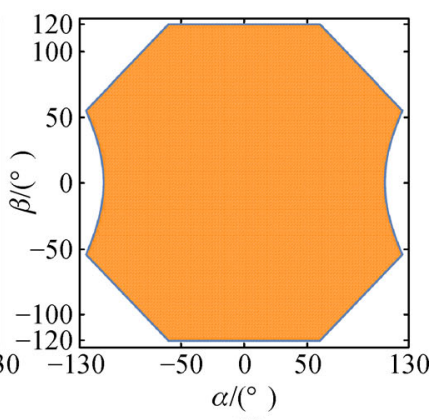

(d)

Fig. 15 The orientation workspace at the position of (a) $z=-240 \mathrm{~mm}$, (b) $z=-180 \mathrm{~mm}$, (c) $z=-120 \mathrm{~mm}$, and (d) $z=60 \mathrm{~mm}$.

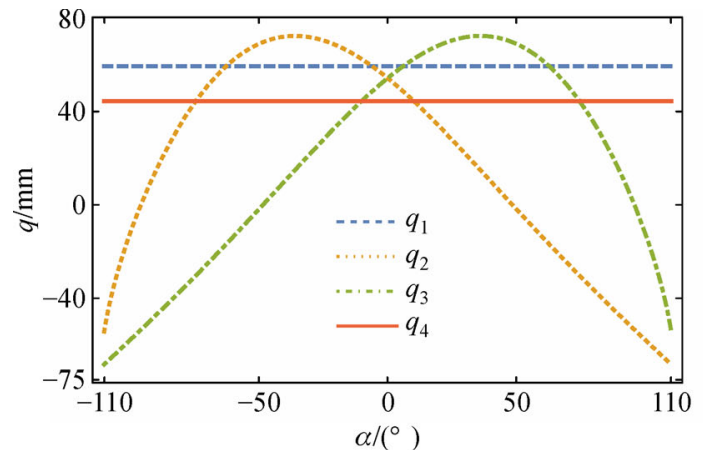

(a)

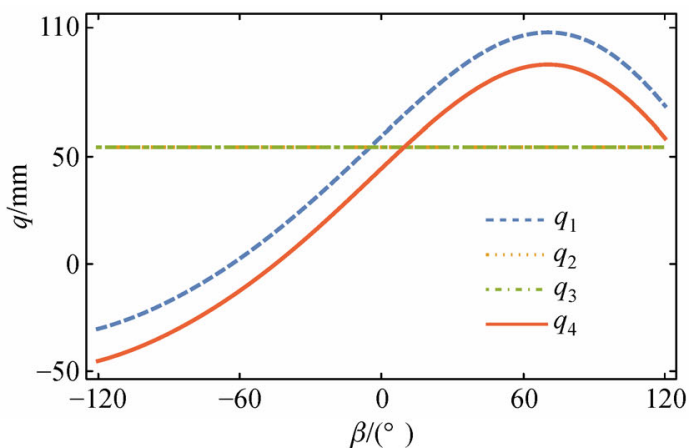

(b)

Fig. 16 Relationships between rotational angles and input parameters: (a) $\alpha$ and $\left(q_{1}, q_{2}, q_{3}, q_{4}\right)$; (b) $\beta$ and $\left(q_{1}, q_{2}, q_{3}, q_{4}\right)$.

analysis of motion/force transmission, dynamics, singularity, stiffness and optimization of the proposed manipulators and the performance comparison with other existed spindle heads.

Acknowledgements The authors gratefully acknowledge the financial support of the National Nature Science Foundation of China (Grant Nos. 51975039 and 51675037) and the Fundamental Research Funds for the Central Universities (Grant No. 2018JBZ007).

Open Access This article is licensed under a Creative Commons Attribution 4.0 International License, which permits use, sharing, adaptation, distribution and reproduction in any medium or format, as long as you give appropriate credit to the original author(s) and the source, provide a link to the Creative Commons licence, and indicate if changes were made.

The images or other third party material in this article are included in the article's Creative Commons licence, unless indicated otherwise in a credit line to the material. If material is not included in the article's Creative Commons licence and your intended use is not permitted by statutory regulation or exceeds the permitted use, you will need to obtain permission directly from the copyright holder.

To view a copy of this licence, visit http://creativecommons.org/licenses/ by $/ 4.0 \%$.

\section{References}

1. Sun T, Yang S F, Huang T, et al. A finite and instantaneous screw based approach for topology design and kinematic analysis of 5-axis parallel kinematic machines. Chinese Journal of Mechanical Engineering, 2018, 31(1): 44

2. Fan C X, Liu H Z, Zhang Y B. Type synthesis of 2T2R, 1T2R and 2R parallel mechanisms. Mechanism and Machine Theory, 2013, 61(1): 184-190

3. Li Q C, Huang Z, Hervé J M. Type synthesis of 3R2T 5-DOF parallel mechanisms using the Lie group of displacements. IEEE Transactions on Robotics and Automation, 2004, 20(2): 173-180

4. Shim J H, Kwon D S, Cho H S. Kinematic analysis and design of a six DOF 3-PRPS in-parallel manipulator. Robotica, 1999, 17(3): 269-281

5. Sofka J, Skormin V, Nikulin V, et al. Omni-Wrist III-A new generation of pointing devices. Part I. Laser beam steering devices - Mathematical modeling. IEEE Transactions on Aerospace and Electronic Systems, 2006, 42(2): 718-725

6. Sofka J, Skormin V, Nikulin V, et al. Omni-Wrist III-A new generation of pointing devices. Part II. Gimbals systems - Control. IEEE Transactions on Aerospace and Electronic Systems, 2006, 42(2): 726-734

7. Company O, Marquet F, Pierrot F. A new high-speed 4-DOF parallel robot synthesis and modeling issues. IEEE Transactions on Robotics and Automation, 2003, 19(3): 411-420

8. Krut S, Benoit M, Ota H, et al. I4: A new parallel mechanism for Scara motions. In: Proceedings of the 2003 IEEE International Conference on Robotics and Automation. New York: IEEE, 2003, 
$1875-1880$

9. Nabat V, Rodriguez M, Company O, et al. Par4: Very high speed parallel robot for pick-and-place. In: Proceedings of the IEEE/RSJ International Conference on Intelligent Robots and Systems (IROS). Washington, D.C.: IEEE Computer Society, 2005, 553-558

10. Guo S, Ye W, Qu H B, et al. A serial of novel four degrees of freedom parallel mechanisms with large rotational workspace. Robotica, 2016, 34(4): 764-776

11. Guo S, Fang Y F, Qu H B. Type synthesis of 4-DOF no overconstrained parallel mechanisms based on screw theory. Robotica, 2012, 30(1): 31-37

12. Wang $C Z$ Z, Fang Y F, Fang H R. Novel $2 R 3 T$ and $2 R 2 T$ parallel mechanisms with high rotational capability. Robotica, 2017, 35(2): 401-418

13. Wang C Z, Fang Y F, Guo S. Design and analysis of $3 R 2 T$ and 3R3T parallel mechanisms with high rotational capability. Journal of Mechanisms and Robotics, 2016, 8(1): 011004

14. Li Q C, Chen Q H, Wu C Y, et al. Geometrical distribution of rotational axes of 3-[R][S] parallel mechanisms. Mechanism and Machine Theory, 2013, 65(7): 46-57

15. Xu L M, Li Q C, Zhang N B, et al. Mobility, kinematic analysis, and dimension optimization of new three-degrees-of-freedom parallel manipulator with actuation redundancy. Journal of Mechanisms and Robotics, 2017, 9(4): 041008

16. Xie F G, Liu X J, Zhou Y H. Development and experimental study of a redundant hybrid machine with five-face milling capability in one setup. International Journal of Precision Engineering and Manufacturing, 2014, 15(1): 13-21

17. Wahl J. US Patent, 6431802B1, 2002-08-13

18. Huang T, Liu H T. PCT Patent, WO2007124637A1, 2007-08-11

19. Sun T, Huo X M. Type synthesis of $1 T 2 R$ parallel mechanisms with parasitic motions. Mechanism and Machine Theory, 2018, 128: 412-428

20. Li Q C, Hervé J M. 1T2R parallel mechanisms without parasitic motion. IEEE Transactions on Robotics, 2010, 26(3): 401-410

21. Ye W, He L Y, Li Q C. A new family of symmetrical 2 T2R parallel mechanisms without parasitic motion. Journal of Mechanisms and Robotics, 2018, 10(1): 011006

22. Wang Y, Yu J J, Pei X. Fast forward kinematics algorithm for realtime and high-precision control of the 3-RPS parallel mechanism. Frontiers of Mechanical Engineering, 2018, 13(3): 368-375

23. Wang L P, Xu H Y, Guan L W. Kinematics and inverse dynamics analysis for a novel 3-PUU parallel mechanism. Robotica, 2016,
35(10): 2018-2035

24. Li Y, Xu Q. A new approach to the architecture optimization of a general 3-PUU translational parallel manipulator. Journal of Intelligent \& Robotic Systems, 2006, 46(1): 59-72

25. Tsai L W. The enumeration of a class of three-DOF parallel manipulators. In: Proceedings of the 10th World Congress on the Theory of Machine and Mechanisms. Oulu, 1999, 1123-1126

26. Yang S F, Sun T, Huang T. Type synthesis of parallel mechanisms having 3T1R motion with variable rotational axis. Mechanism and Machine Theory, 2017, 109: 220-230

27. Zhao T S, Dai J S, Huang Z. Geometric synthesis of spatial parallel manipulators with fewer than six degrees of freedom. Proceedings of the Institution of Mechanical Engineers. Part C, Journal of Mechanical Engineering Science, 2002, 216(12): 1175-1185

28. Rodriguez-Leal E, Dai J S, Pennock G R. Screw-system-based mobility analysis of a family of fully translational parallel manipulators. Mathematical Problems in Engineering, 2013, 2013: 262801

29. Lee C C, Hervé J M. Type synthesis of primitive Schoenflies-motion generators. Mechanism and Machine Theory, 2009, 44(10): 1980 1997

30. Lee C C, Hérve J M. Parallel mechanisms generating 3-DoF finite translation and (2 or 1)-DOF infinitesimal rotation. Mechanism and Machine Theory, 2012, 51(5): 185-194

31. Li Y, Wang L, Liu J F, et al. Applicability and generality of the modified Grübler-Kutzbach criterion. Chinese Journal of Mechanical Engineering, 2013, 26(2): 257-263

32. Fang Y F, Tsai L W. Structure synthesis of a class of 4-DoF and 5-DoF parallel manipulators with identical limb structures. International Journal of Robotics Research, 2002, 21(9): 799-810

33. Ye W, Fang Y F, Zhang K T, et al. A new family of reconfigurable parallel mechanisms with diamond kinematotropic chain. Mechanism and Machine Theory, 2014, 74(6): 1-9

34. Pond G, Carretero J. Architecture optimization of three 3-PRS variants for parallel kinematic machining. Robotics and Computerintegrated Manufacturing, 2009, 25(1): 64-72

35. Li Q C, Chen Z, Chen Q, et al. Parasitic motion comparison of 3PRS parallel mechanism with different limb arrangements. Robotics and Computer-Integrated Manufacturing, 2011, 27(2): 389-396

36. Chen X, Liu X J, Xie F G, et al. A comparison study on motion/force transmissibility of two typical 3-DOF parallel manipulators: The sprint Z3 and A3 tool heads. International Journal of Advanced Robotic Systems, 2014, 11(1): 1-10 